Int Arch Allergy Immunol 1995;107:600

\title{
Subject Index, Vol. 107, 1995
}

For the Subject Index of No. 1-3, please see pp. 470-473.

Aerosol challenge 569

Aging 491

Allergens 557

Allergy 547

Antikeratin antibody 508

Antiperinuclear factor 508

Basophils 587

Betvl 595

Biased T-cell receptor usage 581

Bronchoalveolar lavage lymphocytes 581

CD8+cells 581

Cell binding 484

cycle 491

recruitment 475

Cerebral lupus erythematosus 592 Cerebrospinal fluid filtration 592 Chemokines 475, 541

Collagen 484 Cytokine(s) 491,519

- $\quad$ profiles 498

Dendritic epidermal T cells 498

Eosinophil migration 533

Experimental allergic rhinitis 576

Extracellular matrix 484

Fibroblast 519

Food allergens 566

Ganglioside GM3 527

Glycoprotein 557

Guinea pig 576

Histamine 576, 587

House dust 566

Human 541

IgE antibodies 547

IgE-like antibody 569

Immunocytochemistry 541

Immunoglobulin 519

Immunosuppressive therapy 592

Indoor allergen 569

Inflammation 475

Intercellular adhesion molecule-1 533

Interleukin-3 527,587 
Interleukin-4 527

Kinetics 557

ß-Lactoglobulin 566

Leukotrienes 587

Mast cell(s) 527, 547

MlP-la 541

Mononuclear cell 519

Nasal spray 576

Nomenclature 475

Oligoclonality 581

Oncogenes 491

Ovomucoid 566

Oxatomide 576

Parthenium hysterophorus 557

Passive cutaneous anaphylaxis 569

Peripheral blood lymphocytes 581

- - mononuclear cells 595

Phenotypic heterogeneity 498

Platelet-activating factor 533

Platelets 541

Profilaggrin 508

Proliferative response 595

Protein modules 484

Radioallergosorbent test 595

RANTES 541

Rat 576

RBL 547

Rheumatoid arthritis 508

Scleroderma 519

Self-assembly 484

Self-reactivity 498

Sensitization 566

Signal transduction 491

Storage 541

Summer-type hypersensitivity pneumonitis

$581 \mathrm{~T}$ lymphocytes $491 \mathrm{~T}$-cell receptor $581 \gamma \delta+\mathrm{T}$ cells 498 Valency 547

Vascular cell adhesion molecule-1 533 Vß8 581 